\title{
Enhanced green fluorescent protein expression may be used to monitor murine coronavirus spread in vitro and in the mouse central nervous system
}

\author{
Jayasri Das Sarma, ${ }^{1}$ Esther Scheen, ${ }^{2}$ Su-hun Seo, ${ }^{2}$ Michael Koval, ${ }^{1}$ and Susan R Weiss ${ }^{2}$ \\ Departments of ${ }^{1}$ Physiology and ${ }^{2}$ Microbiology, University of Pennsylvania, School of Medicine, \\ Philadelphia, Pennsylvania, USA
}

\begin{abstract}
Targeted recombination was used to select mouse hepatitis virus isolates with stable and efficient expression of the gene encoding the enhanced green fluorescent protein (EGFP). The EGFP gene was inserted into the murine coronavirus genome in place of the nonessential gene 4. These viruses expressed the EGFP gene from an mRNA of slightly slower electrophoretic mobility than mRNA 4. EGFP protein was detected on a Western blot of infected cell lysates and EGFP activity (fluorescence) was visualized by microscopy in infected cells and in viral plaques. Expression of EGFP remained stable through at least six passages in tissue culture and during acute infection in the mouse central nervous system. These viruses replicated with similar kinetics and to similar final extents as wild-type virus both in tissue culture and in the mouse central nervous system (CNS). They caused encephalitis and demyelination in animals as wild-type virus; however, they were somewhat attenuated in virulence. Isogenic EGFP-expressing viruses that differ only in the spike gene and express either the spike gene of the highly neurovirulent MHV-4 strain or the more weakly neurovirulent MHV-A59 strain were compared; the difference in virulence and patterns of spread of viral antigen reflected the differences between parental viruses expressing each of these spike genes. Thus, EGFP-expressing viruses will be useful in the studies of murine coronavirus pathogenesis in mice. Journal of NeuroVirology (2002) 8, 381-391.
\end{abstract}

Keywords: murine coronavirus; viral genetics; viral pathogenesis

\section{Introduction}

Murine coronavirus mouse hepatitis virus (MHV) infection of the mouse provides a model to study both acute and chronic virus-induced neurological disease

Address correspondence to Susan R Weiss, Department of Microbiology, University of Pennsylvania, School of Medicine, Philadelphia, PA 19104, USA. E-mail: weisssr@mail.med.upenn. edu

This work was supported by NIH grants AI-17418, NS-30606, and NS-21954, and a National Multiple Sclerosis Society grant RG-2585 to SRW, and an American Heart Association Grant-inAid and NIH GM61012 to MK. Jayasri Das Sarma was supported in part by an advanced postdoctoral fellowship, FG1431-A-1, from the National Multiple Sclerosis Society. We thank Ehud Lavi for careful assessment of pathology.

Received 15 May 2002; revised 13 June 2002; accepted 2 July 2002. as well as hepatitis. Intracranial or intranasal inoculation of susceptible mice with neurotropic strains of MHV results in a range of outcomes from acute encephalomyelitis to chronic demyelinating disease (Houtman and Fleming, 1996). Two strains that differ markedly in their neurovirulence are MHV-4 and MHV-A59. Infection of C57BL/6 weanling mice with a low dose of the highly neurovirulent MHV-4 (a strain [JHM] isolate) produces a severe and fatal encephalitis, whereas much higher doses of MHV-A59, a mildly neurovirulent strain, are required to produce a mild encephalitis (Lavi et al, 1984, 1990; Phillips et al, 1999).

We have used targeted recombination to select viruses in which spike genes have either been exchanged between strains (Phillips et al, 1999; Das Sarma et al, 2000; Navas et al, 2001) or mutated at specific sites within the gene. Infection of animals 
with such viruses has allowed us to demonstrate that the spike gene plays a major role in the determining pathogenic outcome, including tissue tropism and virulence. An important aspect of these studies has been to observe the spread of viral antigen throughout the central nervous system (CNS) and quantify the numbers and locations of infected cells as a function of time after infection. This has been carried out by using viral specific antibodies to detect antigen in tissue. Our goal here was to select recombinant viruses with efficient and stable expression of a marker gene, enhanced green fluorescent protein (EGFP), to facilitate the detection of spread of viruses within mice as well as to trace virus entry and spread in cell cultures.

Our approach was to replace a nonessential portion of the MHV genome with the EGFP gene. There are several MHV genes that have been demonstrated to be nonessential for replication in tissue culture. These include ORFs 2a (Schwarz et al, 1990), ORFs 4a, 4b (or ORF4 in some strains) (Yokomori and Lai, 1991), and ORF 5a (Yokomori and Lai, 1991), which all encode small nonstructural proteins as well as the hemagglutinin-esteras e (HE) gene, which is expressed in some isolates of MHV-4, and is a pseudogene in A59 (Luytjes et al, 1988). Recently, it has been shown that abrogation of expression of the JHM (MHV-4) ORF4 by interruption of the open reading frame does not decrease neurovirulence (Ontiveros et al, 2001). Thus, gene 4 was chosen as a location to insert the EGFP gene. In a previous study (Fischer et al, 1997), Masters and colleagues were able to replace gene 4 of MHV-A59 with the GFP gene; however, although mRNA encoding GFP was detected, the expression level of the protein was not sufficiently high to detect the activity by fluorescent microscopy. We used a similar approach with modifications designed to maximize expression levels. We used a modified version of the GFP gene, called enhanced green fluorescent protein (EGFP), which has been optimized for brighter fluorescence (Cormack et al, 1996) and in which codon utilization has been maximized for translation in eukaryotic cells (Haas et al, 1996), and a modified EGFP donor plasmid, which results in selection of recombinant viruses with higher expression levels of the mRNA expressing EGFP.

We report here the expression of the EGFP gene from the position of gene 4 within recombinant viruses that express either the MHV-A59 or the MHV-4 spike gene but are otherwise isogenic. Expression of the EGFP gene is stable over multiple passages in vitro and at a high enough level to readily detect in cultured cells and in the CNS of infected animals. These viruses reflect the pathogenic phenotypes of the parental viruses lacking EGFP. Thus, the use of EGFP as a marker protein promises to be useful in studies of viral spread in cultured cells as well as spread and pathogenesis in the mouse central nervous system.

\section{Results}

Selection of recombinant murine coronavirus expressing the enhanced green fluorescent protein Our approach to selecting a recombinant MHV expressing the marker protein, GFP, was to replace the MHV nonstructural gene 4 with the EGFP gene. As described above, we chose this region of the genome because it had been previously demonstrated that expression of gene 4 is not essential for the replication of MHV in vitro (Yokomori and Lai, 1991), nor is it essential for full neurovirulence of MHV-JHM (Ontiveros et al, 2001). We introduced the EGFP gene into recombinant isogenic viruses differing only in the spike gene; these are $S_{A 59} R$, a recombinant wildtype MHV-A59, and $S_{4} R$, a virus with the MHV-4 spike in place of the A59 spike, with all other genes derived from MHV-A59. The EGFP-expressing viruses were selected as described in Materials and methods. Briefly, targeted recombination was carried out between synthetic capped RNAs transcribed from pMH54 EGFP and pMH54-S4EGFP and the recipient virus $\mathrm{fMHV}$, according to the scheme depicted in Figure 1 and Materials and methods. Viral plaques exhibiting green fluorescence, derived from putative recombinants, were picked and plaque-purified twice more. Two plaque-purified viruses, derived from independent recombination events for each plasmid, were used for further characterization. Recombinant viruses with the $A 59$ spike were named $S_{\text {A59 }} R_{\text {EGFP-1 }}$ and $S_{\text {A59 }} R_{\text {EGFP-2 }}$ and those with the MHV-4 spike were named $S_{4} R_{E G F P-1}$ and $S_{4} R_{E G F P-2}$. Because the two viruses in each pair were identical in all ways we have examined, we will show the data, in most cases, for only one and refer to them as $S_{\text {A59 }} R_{\text {EGFP }}$ and $S_{4} R_{\text {EGFP. (We have shown previously that pairs }}$ of viruses selected this way share all in vitro and in vivo properties examined) (Leparc-Goffart et al, 1998; Phillips et al, 1999). Similarly, parental recombinant viruses $\mathrm{S}_{\mathrm{A} 59} \mathrm{R} 16$ (wild-type MHV-A59) and $\mathrm{S}_{4} \mathrm{R} 22$ (expressing the MHV-4 spike, but otherwise isogenic with $S_{A 59} R 16$ ) will be referred to as $S_{A 59} R$ and $S_{4} R$, respectively.

To confirm the presence of the EGFP gene in the recombinant viruses, reverse transcriptase-polymerase chain reaction (RT-PCR) analysis was performed (Figure 2). A primer pair (IZJ5 and IZJ6; Table 1), spanning the $3^{\prime}$ portion of the $\mathrm{N}$ gene through the $3^{\prime}$ untranslated region, and a primer pair flanking the EGFP gene (pEGFP 5'MetI and pEGFP 3'NotI; Table 1) were used to amplify fragments of 600 and 770 base pairs, respectively, from RNA extracted from cells infected with $S_{\text {A59 }} R_{\text {EGFP }}, S_{4} R_{E G F P}$, as well as parental $\mathrm{S}_{\mathrm{A} 59} \mathrm{R}$ and $\mathrm{S}_{4} \mathrm{R}$ and wild-type MHV-A59 and MHV-4. As expected, the primers in the $\mathrm{N}$ gene amplified a fragment from the RNAs of all viruses, wild-type MHV-A59 and MHV-4, recombinant parental viruses $\mathrm{S}_{\mathrm{A} 59} \mathrm{R}$ and $\mathrm{S}_{4} \mathrm{R}$, as well as EGFP-expressing viruses. The EGFP gene-specific primers amplified only 


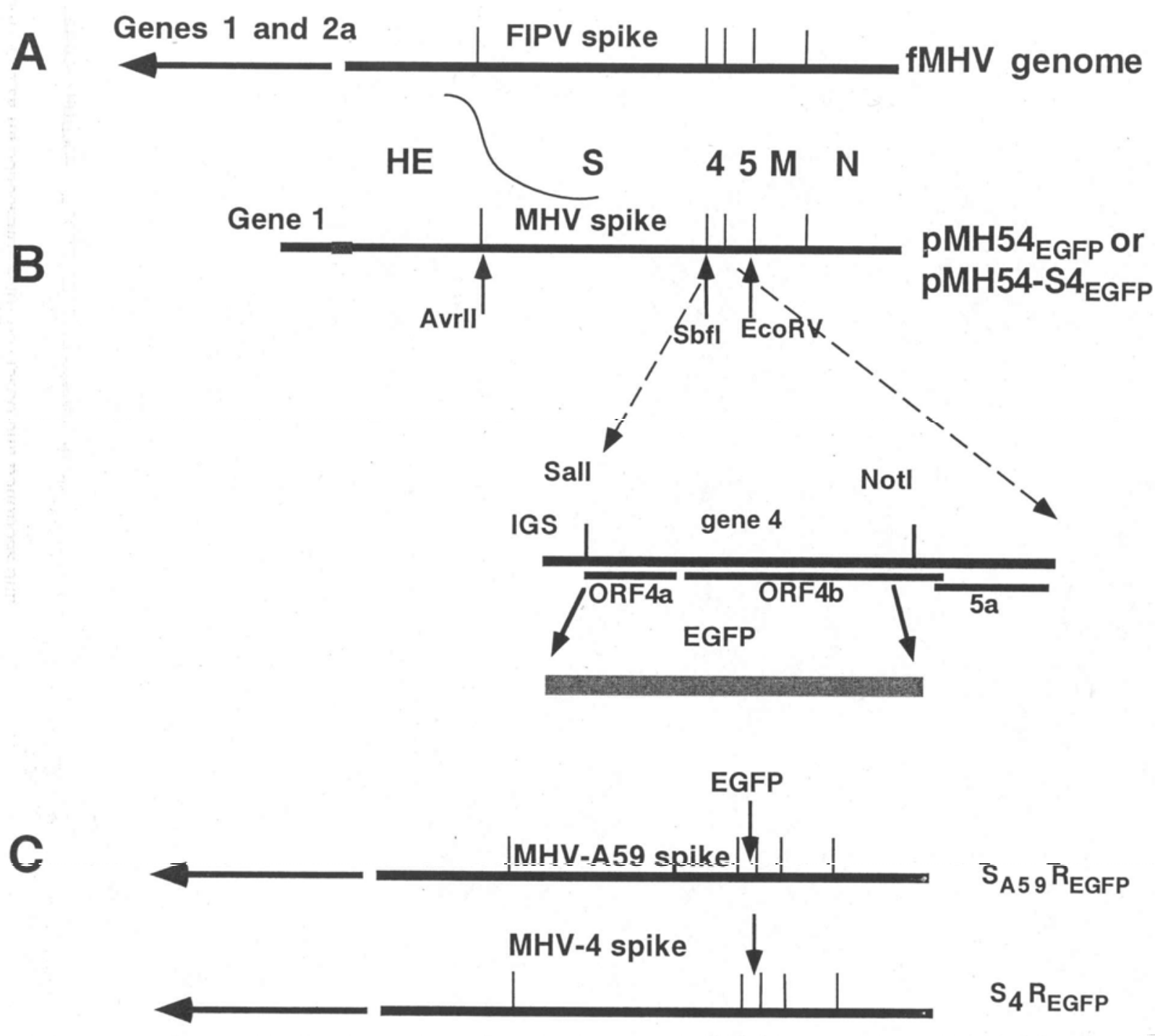

Figure 1 Targeted recombination scheme and the resulting EGFP-expressing viruses. The replacement of ORFs $4 \mathrm{a}$ and $4 \mathrm{~b}$ with the EGFP gene was carried out within the pMH54 and pMH54-S4 plasmids and then introduced into the MHV genome using recombinant technology, all as described in the text. Panel $\mathbf{A}$ is a schematic of the fMHV genome; it encodes the ectodomain of the feline infectious peritonitis spike in the background of the A59 genome. Panel B shows the synthetic RNA transcribed from the vector pMH54EGFP (A59 spike) or pMH54-S4EGFP (MHV-4 spike). The curved line between the genome and the pMH54 RNA indicates the region in which the crossover must have occurred. The restriction sites relevant to the introduction of the EGFP gene are shown. The enlargement of the gene 4 region shows the modifications in which most of ORFs $4 \mathrm{a}$ and $4 \mathrm{~b}$ are replaced by the enhanced green fluorescent protein (EGFP) gene. The IGS (intergenic sequence) is the site of initiation of transcription of mRNA 4. Panel C shows the resulting EGFP-expressing viruses: $\mathrm{S}_{\mathrm{A} 59} \mathrm{R}_{\mathrm{EGFP}}$-expressing the A59 spike (recombinants derived from pMH54 EGFP) and $\mathrm{S}_{4} \mathrm{R}_{\mathrm{EGFP}}$-expressing the MHV-4 spike (recombinants derived from pMH54-S4EGFP).

from the two EGFP-expressing viruses $\mathrm{S}_{\mathrm{A} 59} \mathrm{R}_{\mathrm{EGFP}}$ and $\mathrm{S}_{4} \mathrm{R}_{\mathrm{EGFP}}$, confirming that the EGFP gene was indeed present in these genomes and was of the expected size.

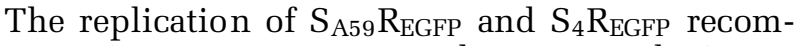
binant viruses were compared to parental viruses $\mathrm{S}_{\mathrm{A} 59} \mathrm{R}$ and $\mathrm{S}_{4} \mathrm{R}$, respectively, by carrying out one-step growth curves in L2 cells. As shown in Figure 3, all viruses replicated efficiently. $S_{\text {A59 }} R_{\text {EGFP replicated }}$ with similar kinetics, and to a similar final extent, as $\mathrm{S}_{\mathrm{A} 59} \mathrm{R}$. As previously observed, viruses expressing the spike gene of MHV-4 $\left(\mathrm{S}_{4} \mathrm{R}\right.$ and $\left.\mathrm{S}_{4} \mathrm{R}_{\mathrm{EGFP}}\right)$ repli- cated to lower final titers than viruses expressing the A59 spike protein $\left(S_{A 59} R\right.$ and $\left.S_{A 59} R_{E G F P}\right) . S_{4} R_{E G F P}$ replicated to similar final titer as parental $S_{4} R$ but displayed slower kinetics. EGFP-expressing viruses induced cell-to-cell fusion as parental viruses (see below, Figure 6).

\section{Expression of EGFP $m R N A$ and protein by} recombinant viruses in vitro

We examined the mRNA patterns from EGFPexpressing recombinant viruses by carrying out Northern blots of RNA extracted from cells infected 


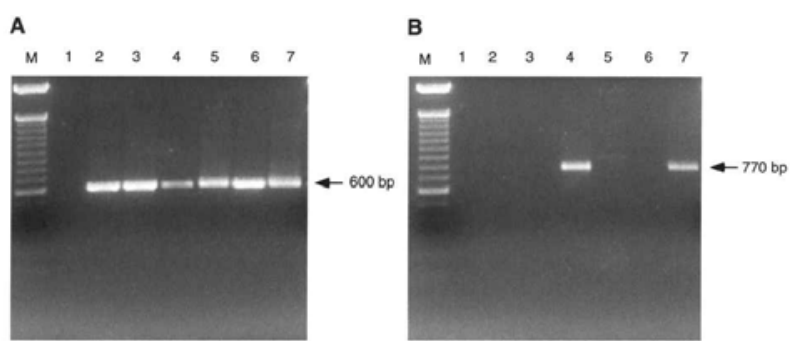

Figure 2 RT/PCR analysis of putative recombinant viruses. Cytoplasmic RNA was extracted from infected L2 cells. A 600nucleotide fragment spanning the nucleocapsid gene and the 3 nontranslated region was amplified using primers IJZ5 and IJZ6 (panel A) or a 770-nucleotide fragment containing the EGFP gene was amplified using primers 5'MetI and 3'NotI (panel B). Fragments were analyzed following agarose gel electrophoresis. Lane 1, mock infected; lane 2, wild type MHV-A59; lane 3, recombinant $\mathrm{S}_{\mathrm{A} 59} \mathrm{R}$; lane 4, recombinant $\mathrm{S}_{\mathrm{A} 59} \mathrm{R}_{\mathrm{EGFP}}$; lane 5, wild-type MHV-4; lane 6, recombinant $S_{4} R$; lane $7, S_{4} R_{E G F P}$. The mobilities of the 100-base pair DNA ladder marker DNAs are indicated.

with parental and EGFP-expressing virus (Figure 4). Total cellular RNAs prepared from mock-, MHVA59-, $S_{\text {A59 }}$ R-, and $S_{\text {A59 }} R_{\text {EGFP-infected cells were elec- }}$ trophoresed in denaturing agarose gels, blotted onto nylon membranes, and hybridized with radiolabeled probes specific for either for the N gene or the EGFP gene (see Materials and methods). As expected, the $\mathrm{N}$ gene probe detected all seven MHV RNAs in cells infected by all viruses. Also as expected, the EGFP probe detected mRNAs 1 to 4 , those containing the EGFP gene only in RNA from cells infected with EGFP-expressing virus (Figure 4). Although mRNAs 5 to 7 of all viruses were identical in size, mRNA 4 of EGFP-expressing viruses migrated more slowly than the corresponding mRNAs from wild-type viruses. Interestingly, the levels of mRNA 4 were consistently higher in recombinant viruses as compared with wild-type parental MHV-A59 (compare lanes 2, 3 , and 4 , Figure 4). Although mRNA 4 was barely detectable in RNA from MHV-A59-infected cells (lane 2), it was abundant in RNA from either $S_{A 59} R-$ or $S_{\text {A59 }} R_{E G F P}$-infected cells (lanes 3 and 4 ). This phenomenon was observed previously with recombinant MHV-4 viruses (Ontiveros et al, 2001); the increased

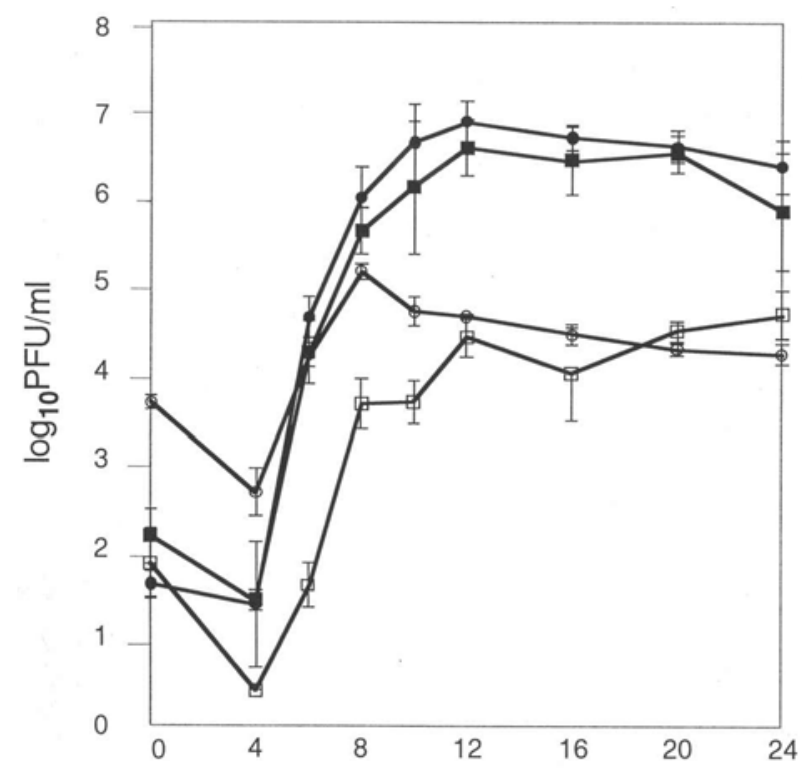

hours post infection

Figure 3 Time course of recombinant virus production in L2 cell culture. $\mathrm{L} 2$ cells were infected in (triplicate) duplicate with $\mathrm{S}_{\mathrm{A} 59} \mathrm{R}$ (ם), $S_{\text {A59 }} R_{\text {EGFP }}(\square), S_{4} R(\bullet), S_{4} R_{E G F P}(0)$ at an MOI of 1 PFU/cell. At the indicated times, cells and media above them were freezethawed 3 times, clarified, and virus titers were determined by plaque assay. Each point represents the mean titer of duplicate samples.

synthesis of mRNA 4 in the recombinant viruses resulted from the three nucleotide changes that were made in pMH54 in order to create an SbfI (Sse8387I) restriction site downstream of the S gene (see Materials and methods). The reason for the transcriptional up-regulation caused by these substitutions is currently unclear, but is likely helpful in achieving the desired level of EGFP expression.

We next investigated the level of EGFP protein expression using immunoblots (Figure 5). Protein lysates from cells infected with parental and EGFPexpressing recombinant viruses (as well as mockinfected cells) were analyzed by polyacrylamide gel electrophoreses, blotted onto polyvinyldene fluoride (PVDF) membranes and probed with anti-EGFP

Table 1 Oligonucleotide primers used for plasmid construction and for sequencing

\begin{tabular}{|c|c|c|c|}
\hline Name & Sequence $\left(5^{\prime} \text { to } 3^{\prime}\right)^{\mathrm{a}}$ & Polarity & Positions (nucleotide in A59 ORFs) \\
\hline IJZ5 & GCTCCAACAGTTGGTGCC & Positive & 890-908 N gene \\
\hline IJZ6 & ACGTAGGACCTTGCTAACTTC & Negative & $166-186$ ( $3^{\prime}$ nontranslated region) \\
\hline pEGFP 5'MetI & TATATCCCACCGGTCGCCACCCTGGTGAGCAAG & Positive & Plasmid sequences flanking EGFP \\
\hline pEGFP $3^{\prime}$ NotI & CTAGAGTCgcggccgcTT TACTTGTACAGCTCG & Negative & Plasmid sequences flanking EGFP \\
\hline FIJ81 & AGTATGGAGGACACCAGGACAG & Positive & $3917-3938$ of S gene \\
\hline RIJ84 & ACCATGCATCACTCACATGCC & Negative & 26-45 (ORF 5a) \\
\hline FIJ83 & TCATCTTAATTCTGGTCGTCC & Positive & $329-349$ ORF $4 b$ \\
\hline RIJ86 & GCTGCTTACTCCTATCATACA & Negative & 173-193 ORF 5b \\
\hline SalI & GGCCCCGTAAGAGGCAgtcgacGC TATGGCCGTGTTGG & Positive & Upstream and adjacent to ORF4a \\
\hline NotI & CTTGGTACTCCAAgcggccgcG ATTATAGTACATTTGGG & Negative & 198-237 ORF $4 \mathrm{~b}$ \\
\hline
\end{tabular}

${ }^{\mathrm{a}}$ Lower case letters indicate restriction enzyme recognition sites. 


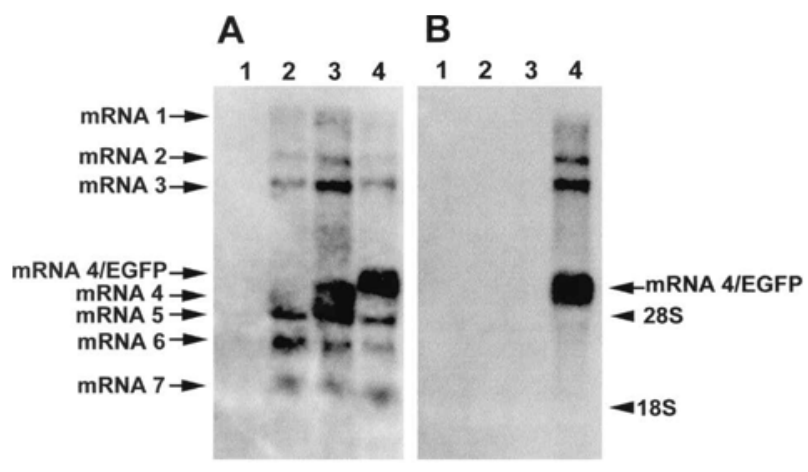

Figure 4 Viral mRNA expression in cells infected with parental and EGFP-expressing viruses. Cytoplasmic RNA was extracted from infected L2 cells; RNA (12 $\mu \mathrm{g} /$ lane) were electrophoresed in $1 \%$ agarose gels, in the presence of formaldehyde. Gels were blotted onto nylon membranes and probed with radiolabeled probes representing the nucleocapsid gene (panel A) and the EGFP gene (panel B). Lane 1, mock infected; lane 2, MHV-A59; lane 3, $\mathrm{S}_{\mathrm{A} 59} \mathrm{R} 16$; lane $4, \mathrm{~S}_{\mathrm{A} 59} \mathrm{R}_{\mathrm{EGFP}}$. Viral mRNAs 1-7 and mRNA 4/EGFP are indicated, as well as the migration of marker $18 \mathrm{~S}$ and $28 \mathrm{~S}$ cellular ribosomal RNAs. (MHV RNA 7 appears to be less abundant than expected. This is probably because the gel was loaded with samples containing enough RNA to assure visualization of the larger, less abundant viral RNAs; the large amount of $18 \mathrm{~S}$ ribosomal RNA present in these samples limits the binding of viral RNA 7 to the blot.)

serum (Molecular Probe, Engene, OR) and in parallel with a monoclonal antibody directed against viral nucleocapsid (N) protein (Materials and methods). As expected, the anti-N antibodies recognized a $50-\mathrm{kDa}$ band in lysates from cells infected with all viruses. (As has been observed previously [Bond et al, 1979], the N protein of wild-type MHV-4 [lane 5] has slightly slower mobility than that of MHV-A59 or the recombinants, which all have the MHV-A59 N gene.) The anti-GFP antiserum recognized a polypeptide migrating at about $31 \mathrm{kDa}$ in lysates from cells infected with EGFP-expressing viruses. Although the calculated molecular mass for monomeric EGFP is $27 \mathrm{kDa}$,

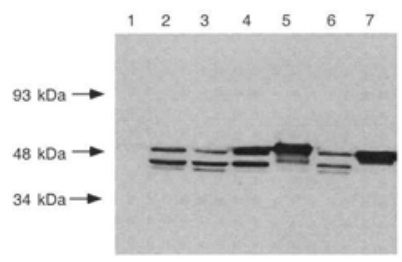

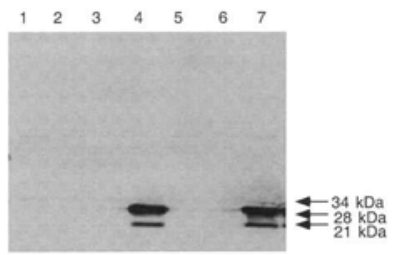

Figure 5 EGFP-expression in cells infected with parental and EGFP-expressing viruses. Lysates of infected L2 cells (25 $\mu \mathrm{g}$ protein/lane) were electrophoresed in 10\% SDS polyacrylamide gels. Proteins were transferred onto PVDF membranes and probed with anti-nucleocapsid monoclonal antibody (panel A) or rabbit anti-EGFP antiserum (panel B), followed by HRP-conjugated secondary antisera and then visualized by chemiluminescence (all as described in Materials and methods). Lane 1, mock infected; lane 2, wild-type MHV-A59; lane 3, recombinant $\mathrm{S}_{\mathrm{A} 59} \mathrm{R}$; lane 4, $S_{A 59} R_{E G F P}$; lane 5, wild-type MHV-4; lane 6, recombinant $S_{4} R$;

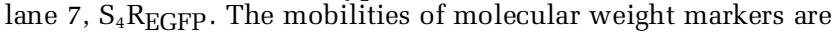
indicated.

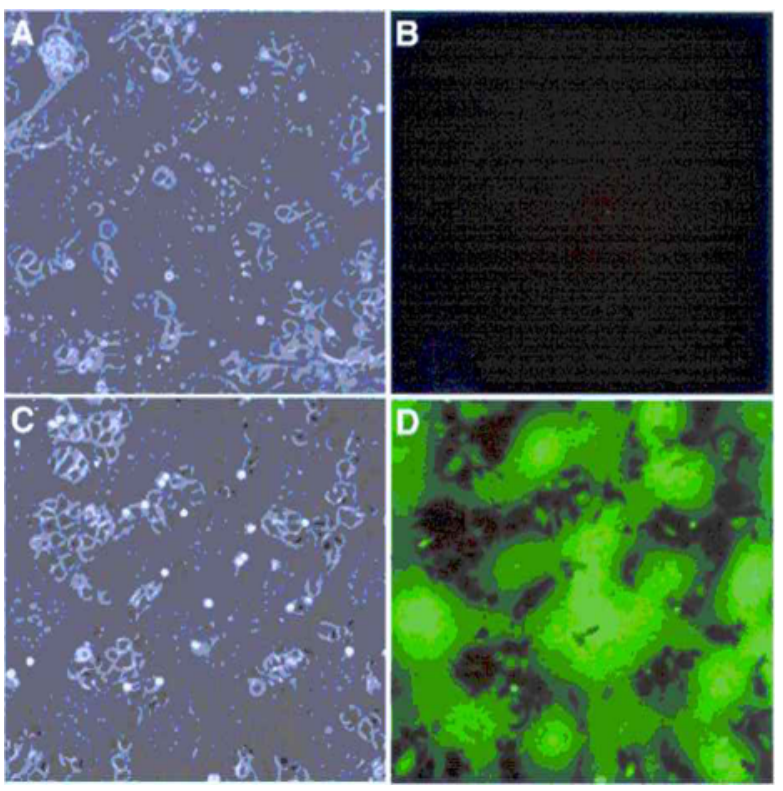

Figure 6 EGFP-expression in vitro in cells. L2 cells were infected in duplicate with $\mathrm{S}_{\mathrm{A} 59} \mathrm{R}(\mathbf{A}, \mathbf{B})$ or $\mathrm{S}_{\mathrm{A} 59} \mathrm{R}_{\mathrm{EGFP}}(\mathbf{C}, \mathbf{D})$ at an MOI of $1 \mathrm{PFU} / c e l l$. At $12 \mathrm{~h}$ post infection, cells were observed by phase microscopy (A, C) and for EGFP fluorescence (B, D).

the observations of a slightly slower electrophoretic mobility and the presence of other less prominent bands have been reported previously (Fischer et al, 1997).

In order to be useful as a marker for infected cells, it was important that EGFP was expressed at a high enough level to readily detect by microscopy. Thus we examined murine L2 cells infected with either $\mathrm{S}_{\mathrm{A} 59} \mathrm{R}$ or $\mathrm{S}_{\mathrm{A} 59} \mathrm{R}_{\mathrm{EGFP}}$ at $12 \mathrm{~h}$ post infection, by both phase contrast and fluorescent microscopy (Figure 6). Similar levels of syncytia were observed in cells infected with either virus. However, EGFP fluorescence is observed within the syncytia of cells infected with $S_{\text {A59 }} R_{\text {EGFP. The }}$ absence of fluorescence in $S_{A 59} R$ infected cells shows the specificity of the fluorescence for EGFP.

\section{Pathogenesis of viruses expressing EGFP and either} the A59 or MHV-4 spike genes

Although it has been shown that expression of gene 4 was not necessary for full neurovirulence of MHV-4, this was demonstrated by introducing mutations within the gene rather than replacing the gene and altering the size of the gene (Ontiveros et al, 2001). Thus, we investigated whether replacement of the EGFP gene for gene 4 may effect virulence. The virulence of the EGFP-expressing recombinant viruses was compared to that of parental recombinants $S_{A 59} R$ and $\mathrm{S}_{4} \mathrm{R}$. Intracranial lethal dose $(\mathrm{LD})_{50}$ measurements revealed that both EGFP-expressing recombinant viruses, $S_{A 59} R_{E G F P}$ and $S_{4} R_{E G F P}$, were less virulent; the $\mathrm{LD}_{50}$ values were about 10 -fold greater for EGFP-expressing viruses, as compared with their corresponding wild-type recombinant viruses, $S_{A 59} R$ 


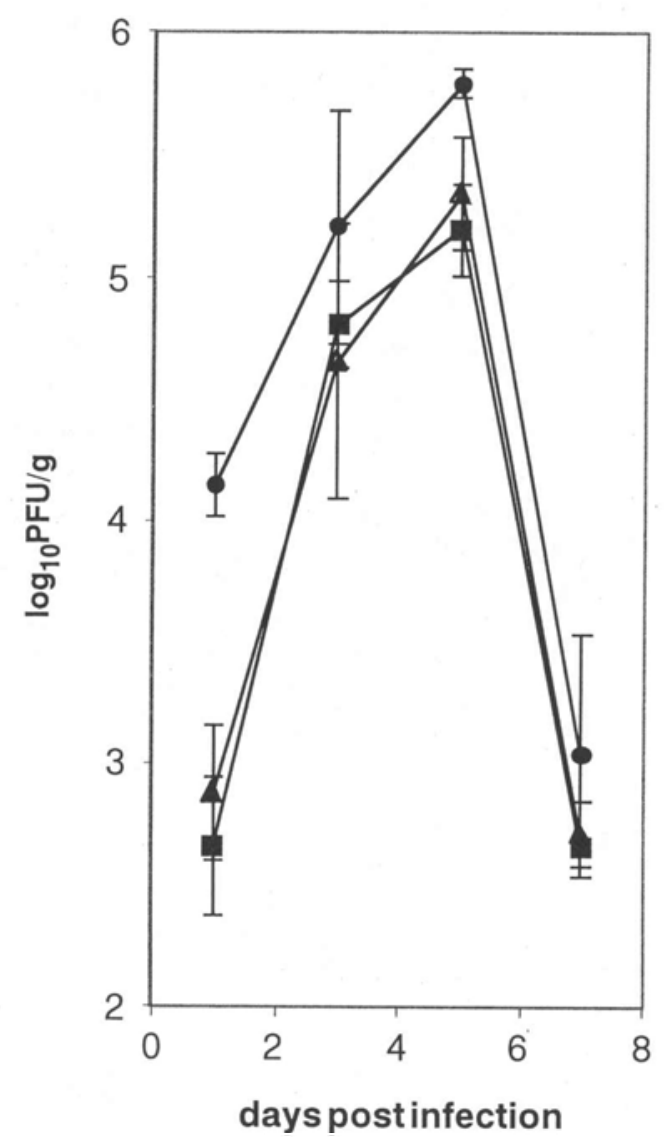

Figure 7 Viral replication in the brains of animals following intracranial inoculation. C57BL/6 weanling mice were infected with 10,000 PFU of $\mathrm{S}_{\mathrm{A} 59} \mathrm{R}(\bullet)$ and two clones of EGFP-expressing virus $\mathrm{S}_{\mathrm{A} 59} \mathrm{R}_{\mathrm{EGFP}-1}(\boldsymbol{\square})$ and $\mathrm{S}_{\mathrm{A} 59} \mathrm{R}_{\mathrm{EGFP}-1}(\boldsymbol{\Delta})$. Animals were sacrificed at the indicated times, and virus titers in the brains were determined by plaque assay. The data shown represent the means (and standard deviations) of the titers from five animals. The difference in titers between $\mathrm{S}_{\mathrm{A} 59} \mathrm{R}$ and either $\mathrm{S}_{\mathrm{A} 59} \mathrm{R}_{\mathrm{EGFP}-1}$ or $\mathrm{S}_{\mathrm{A} 59} \mathrm{R}_{\mathrm{EGFP}-2}$ were statistically different at days 1 and 5 post infection, using the Wilcoxon rank sum $W$ test; $P=.009$ for each pair of viruses.

and $S_{4} R$. Thus, relative levels of virulence of the A59 spike- and MHV-4 spike-expressing viruses were similar to the relative levels of the parental viruses.

In order for these EGFP-tagged viruses to be useful in studies of viral pathogenesis, we needed to verify that they replicated efficiently in the CNS and caused a similar disease, acute encephaliti s (and, in addition, hepatitis in the case of $S_{\mathrm{A} 59} \mathrm{R}_{\mathrm{EGFP}}$ ), as well as chronic demyelinating disease, as parental viruses. Thus, we carried out intracranial inoculation s of two independently isolated, clones of $S_{\text {A59 }} R_{\text {EGFP }}\left(S_{\text {A59 }} R_{\text {EGFP-1 }}\right.$ and $\left.\mathrm{S}_{\mathrm{A} 59} \mathrm{R}_{\mathrm{EGFP}-2}\right)$ and $\mathrm{S}_{\mathrm{A} 59} \mathrm{R}$ at $10^{4}$ plaque-forming unit (PFU)/mouse and measured virus replication in the brains as a function of time after infection. The kinetics and the final viral titers were similar, albeit slightly lower, for the EGFP-expressing viruses compared to wild-type virus; the peak of viral replica- tion was at day 5 for all viruses, as observed previously (Figure 7) (Phillips et al, 1999). (The differences in titer observed between $S_{\mathrm{A} 59} R$ and $S_{\text {A59 }} R_{E G F P-1}$ or $\mathrm{S}_{\mathrm{A} 59} \mathrm{R}_{\mathrm{EGFP}-2}$ were statistically significantly at days 1 and 5.) We carried out similar infections with $S_{4} R$ and $S_{4} R_{\text {EGFP }}$ in which we titered virus from brains at 5 days post infection. Similar to the result obtained for $S_{A 59} R$ and $S_{A 59} R_{E G F P}, S_{4} R_{E G F P}$ replicated to a titer approximately 10 -fold less than $S_{4} R$. We had learned from previous studies that the level of infectious virus is not necessarily a predictor of virulence (Phillips et al, 1999); thus we carried out further comparisons of spread of these viruses.

We had shown previously that $\mathrm{S}_{\mathrm{A} 59} \mathrm{R}$ and $\mathrm{S}_{4} \mathrm{R}$, isogenic viruses differing only in the spike gene, displayed similar regional localization of antigenpositive cells, but different numbers of infected cells (Phillips et al, 1999); thus, the high neurovirulence of $\mathrm{S}_{4} \mathrm{R}$ was associated with larger numbers of infected cells. In order to determine whether this difference in spread of viral antigen was also a property for the EGFP-expressing viruses derived from $S_{\text {A59 }} R$ and $S_{4} R$, we compared the spread of $S_{\text {A59 }} R_{E G F P}$ and $S_{4} R_{E G F P}$ in the CNS. Frozen sagittal brain sections, from the same animals described above, were postfixed and examined for EGFP fluorescence by microscopy as described in Materials and methods. EGFP was detected in discrete foci in the same regions of the brain in mice infected by both EGFPexpressing viruses as reported previously for parental viruses $S_{A 59} R$ and $S_{4} R$. (We examined at least three sections from two animals infected with each virus.) There were no detectable differences in the regional location of viral antigen expression in the brain (Phillips et al, 1999). Areas of viral antigen-positive cells for both viruses included the olfactory bulbs, mid brain, cortex, subiculum, basal forebrain structures, and regions of the brainstem. Figure 8 shows an example of a portion of sagittal sections cut from the middle of the brains of animals infected with $S_{A 59} R_{E G F P}$ and $S_{4} R_{E G F P}$ at the peak of viral antigen expression, day 5. Despite the similarity in regional localization in the brain, $\mathrm{S}_{4} \mathrm{R}$ clearly exhibited a significantly larger number of viral antigen-positive cells. When quantified (as described in Materials and methods), the area expressing EGFP fluorescence was 10fold greater in the section from a $S_{4} R_{E G F P}$-infected mouse as compared to that from a $S_{\mathrm{A} 59} R_{\mathrm{EGFP}}$-infected mouse. Figure 8 also shows higher magnification of one region from each section; this illustrates the intracellular localization of the fluorescence.

We demonstrated previously that, along with increased numbers of viral antigen-positive cells, the brains of $\mathrm{S}_{4} \mathrm{R}$-infected animals also displayed higher levels of inflammatory response than brains from $S_{\mathrm{A} 59} \mathrm{R}$-infected mice, while appearing in the same regions in which viral antigen was detected (Phillips et al, 1999). Thus, we examined the inflammation in the brains of the same animals, at day 5, described above for EGFP detection. Thus, 


\section{A

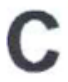

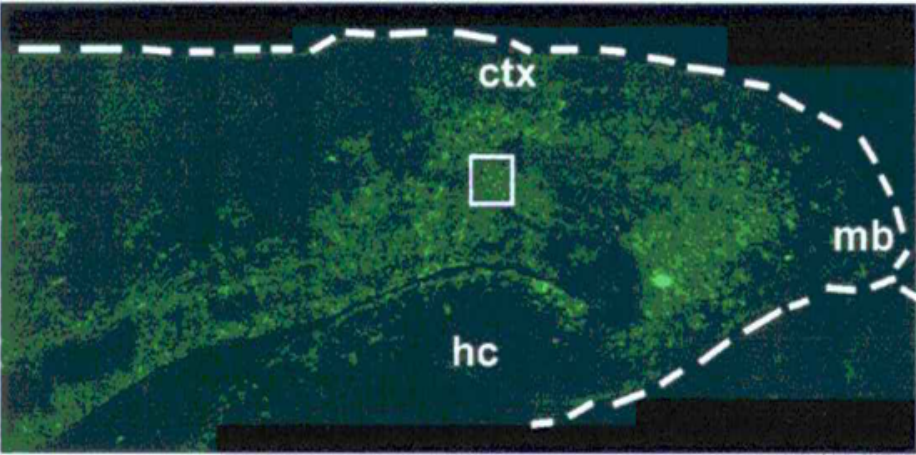
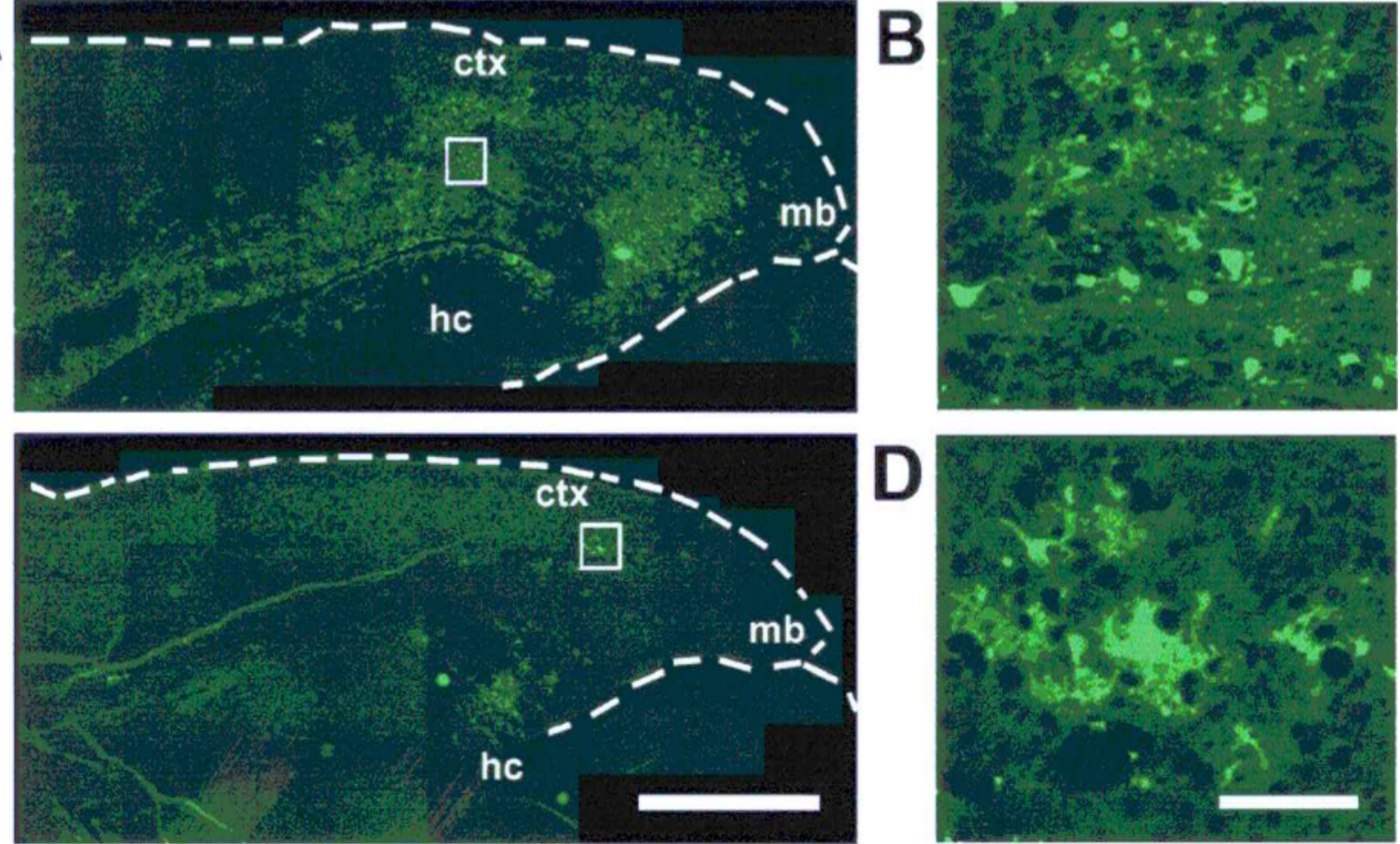

Figure 8 Viral antigen distribution in the CNS of animals infected with EGFP-expressing viruses. C57BL/6 weanling mice were infected

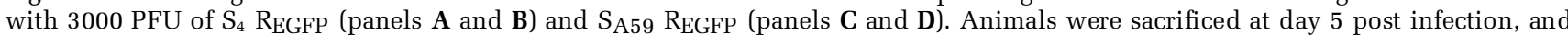
the brains were processed, sectioned, and observed for fluorescence, all as described in Materials and methods. In panels $\mathbf{A}$ and $\mathbf{C}$, the bar represents $2.5 \mathrm{~mm}$; the sections are labeled to orient the section. ctx, cortex; mb, midbrain; hc, hippocampus. Panels $\mathbf{C}$ and $\mathbf{D}$ are enlargements of the boxed areas in panels $\mathbf{A}$ and $\mathbf{C}$; the bar represents $0.25 \mathrm{~mm}$.

additional sections from the same animals analyzed above for viral antigen, infected with 3000 PFU of $S_{\mathrm{A} 59} R_{\mathrm{EGFP}}$ and $\mathrm{S}_{4} \mathrm{R}_{\mathrm{EGFP}}$, were stained with hematoxylin and eosin (H\&E) and analyzed by light microscopy. Brains of animals infected with $\mathrm{S}_{4} \mathrm{R}_{\mathrm{EGFP}}$ revealed moderate encephalitis at day 5 , whereas those infected with $S_{\text {A59 }} R_{E G F P}$ had mild encephalitis. The regions in which inflammation was observed were the same as those positive for viral antigen and similar to those previously observed for parental viruses (Phillips et al, 1999).

Infection of 4-week-old mice with recombinant MHV-A59 $\left(\mathrm{S}_{\mathrm{A} 59} \mathrm{R}\right)$ as well as wild-type MHV-A59 caused chronic spinal cord demyelination; the peak of demyelination was at about 30 days postinfection (Sutherland et al, 1997; Matthews et al, 2001). We measured demyelination in mice infected with $\mathrm{S}_{\mathrm{A} 59} \mathrm{R}$ or $S_{A 59} R_{E G F P}$ at 30 days. At dose of approximately 1 $\mathrm{LD}_{50}\left(5 \times 10^{4}\right.$ PFU), nearly all mice (9 of 10) infected with $S_{\text {A59 }} R_{\text {EGFP }}$ demonstrated demyelination, with an average of $40 \%$ of the quadrants involved. All of the $\mathrm{S}_{\mathrm{A} 59} \mathrm{R}$-infected animals (3 of 3) exhibited demyelination, with the average of $58 \%$ of the spinal cord quadrants involved. The levels of demyelination observed for $S_{A 59} R_{E G F P}$-infected mice are well within the wide range of values observed with $S_{\text {A59 }} R$-infected mice.

We also assessed animals infected with EGFPexpressing viruses for viral replication in the liver and hepatitis. Animals infected with $\mathrm{S}_{\mathrm{A} 59} \mathrm{R}_{\mathrm{EGFP}}$ demonstrated hepatitis as evidenced by viral antigen expression and inflammation in the livers and replication of virus in the liver. Surprisingly, hepatitis was also observed in animals infected with $\mathrm{S}_{4} \mathrm{R}_{\mathrm{EGFP}}$. This is likely due to the very high levels of virus inoculated into animals compared to previous studies, as it was also observed in animals infected with parental $\mathrm{S}_{4} \mathrm{R}$.

Expression of EGFP is stable during passage in vitro and in vivo

In order to assess the stability of the EGFP gene in

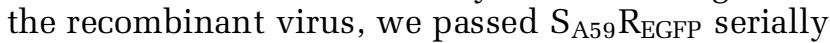
at a multiplicity of infection (MOI) of approximately 1 PFU/cell in L2 cells and examined plaques for EGFP fluorescence at each passage. Nearly all of the plaques were positive for EGFP expression during at least six serial in vitro passages. Virus was plaqued from brain and liver homogenates from animals infected with

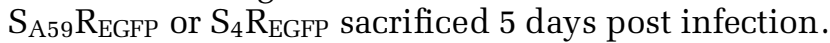
EGFP fluorescence was observed in nearly all of the plaques recovered from the brain. Thus, EGFP activity was stable in recombinant viruses through at least six passages in vitro and it was maintained during infection of the CNS. Interestingly, plaques recovered from the liver were mixed, with the majority negative for EGFP fluorescence. 


\section{Discussion}

We have selected and characterized recombinant murine coronaviruses that express EGFP. This is the first demonstration of stable and efficient expression of a foreign gene from a murine coronavirus genome. We have been able to use EGFP activity to trace the spread of viral antigen both in vitro and in vivo; furthermore, viruses expressing EGFP and either the A59 or MHV-4 spike proteins reflect the properties of the parental viruses, demonstrating that EGFP-tagged viruses will be useful in pathogenesis studies.

Our approach to expressing EGFP from the MHV genome was to select recombinant viruses in which nonstructural gene 4 has been replaced with the EGFP gene. We used this strategy because gene 4 is not essential for replication in tissue culture (Yokomori and Lai, 1991) nor for wild-type levels of neurovirulence (Ontiveros et al, 2001). Furthermore, Fischer et al (1997) had been able to introduce the GFP gene into gene 4 and observe transcription of an mRNA encoding GFP and to detect the protein by Western blot; however, this recombinant virus was not useful for studies of viral spread because the levels of GFP expression were not high enough to detect by fluorescence microscopy.

We were able to achieve a significantly higher level of expression than that observed by Fischer et al (1997), enough to easily detect by fluorescence microscopy, both in infected cells in culture and in mice. This increased activity was a result of two factors. First, we used a modified GFP gene, EGFP (Clontech catalogue 6085-1) that encodes a red-shifted variant of wild-type GFP and has been optimized for brighter fluorescence (Cormack et al, 1996) and in which codon utilization has been maximized for translation in mammalian cells (Haas et al, 1996). The second factor is an unexplained increase in transcription of mRNA 4 that occurs when recombinant viruses are selected using derivatives of plasmid pMH54 in which an SbfI site has been introduced (by silent mutations) into the region between the spike gene and gene 4 (Figure 1). This phenomenon, previously reported by Ontiveros et al (2001), can be observed in the comparison of expression of mRNA 4 from wild-type viruses A59 and MHV-4 and all of the recombinant viruses whether or not they express EGFP (Figure 4). We amplified and sequenced the $5^{\prime}$ end of the mRNA4/EGFP from $\mathrm{S}_{\mathrm{A} 59} \mathrm{R}_{\mathrm{EGFP}}$. This consensus sequence contained the $5^{\prime}$ MHV-A59 leader sequence, followed by the intergenic sequence between spike and gene 4, followed by the EGFP gene. This was consistent with transcription of one major mRNA in the region of mRNA 4; we did not detect additional mRNAs encoding the EGFP gene, as was reported in a previous study (Fischer et al, 1997).

We investigated whether expression of the EGFP gene was stable over many passages of virus in vitro and also during replication in mice. This was of concern, as the EGFP-expressing viruses replicate slightly less efficiently as parental viruses and show some attenuation in virulence in mice; we, thus, reasoned that replication in vitro and or in mice may select for virus in which the EGFP gene has been deleted. Nearly all the plaques examined over six passages of virus in tissue culture retain EGFP fluorescence. Furthermore, nearly all plaques isolated from the brains of infected mice at 5 days post infection are still expressing EGFP. Thus, expression of the EGFP gene remains stable through in vitro passage and during acute infection in the murine CNS. Interestingly, the majority of plaques recovered from the liver during actue infection were negative for EGFP activity. A similar organ-specific loss of a foreign sequence was reported by Slifka et al (2001). In this study, insertion of a foreign sequence from lymphocytic choriomeningitis virus (LCMV) into a recombinant coxsackievirus resulted in a virus that frequently deleted the LCMV sequence after inoculation into animals; the foreign sequences were lost more frequently in virus isolated from the heart whereas viruses isolated from the pancreas were more likely to retain the LCMV sequence (Slifka et al, 2001).

The EGFP-expressing MHV isolated did display somewhat attenuated phenotypes, compared to parental viruses in vivo. This is not due to the disruption of gene 4, as it has been demonstrated that inactivation of gene 4 expression by the introduction of three nucleotide substitutions does not reduce replication of MHV-JHM in vitro nor does it reduce neurovirulence (Ontiveros et al, 2001). Thus it is likely that insertion of the EGFP gene (which is longer than ORF 4) confers a disadvantage to the virus, possibly because the EGFP gene alters the local structure of the RNA, thereby causing a slight disadvantage in replication that results in reduction in pathogenesis. As shown in Figure 7, there is a small but significant difference in replication of $S_{A 59} R_{E G F P}$, as compared with $\mathrm{S}_{\mathrm{A} 59} \mathrm{R}$, in the mouse CNS. It is also possible, but less likely, that expression of EGFP itself may attenuate the EGFP-expressing viruses.

We have tested the utility of EGFP-expressing viruses for in vivo studies by selecting EGFPexpressing viruses that are isogenic except for the spike gene. We know from past studies that otherwise isogenic viruses expressing either the MHV-4 or A59 spike genes differ greatly in their neurovirulence, with the MHV-4 spike-expressing viruses $\left(\mathrm{S}_{4} \mathrm{R}\right)$ displaying very high neurovirulence and an $\mathrm{LD}_{50}$ approximately 1000 -fold lower than the mildly neurotropic A59 spike-expressing viruses $\left(\mathrm{S}_{\mathrm{A} 59} \mathrm{R}\right)$ (Phillips et al, 1999). Thus, we compared such viruses tagged with EGFP in infection and spread of virus in the CNS. EGFP-expressing viruses, expressing either the MHV-4 or A59 spike proteins, replicate efficiently in the CNS and induce encephalitis. At least in the case of $\mathrm{S}_{\mathrm{A} 59} \mathrm{R}_{\mathrm{EGFP}}$, demyelination was also detected. (We did not examine demyelination for $S_{4} R_{E G F P .)}$ The spread of the 
EGFP-expressing viruses within the CNS was similar to that reported for the parental viruses. Encephalitis was focal, with similar regions of involvement as previously reported (Phillips et al, 1999). The major difference in viral antigen in the CNS for both parental and EGFP-expressing viruses is in the number of antigen-positive cells.

A potential use for EGFP-expressing viruses is to use fluorescence to follow virus spread over time without having to terminate infection by fixation and detection of viral antigen by immunofluorescence. This will be of use in cultured cells to monitor viral entry and to observe spread of virus by time-lapse microscopy using living cells. We have recently begun to examine virus spread over time in primary neuronal cultures. In a recent study (Smith et al, 2000), an EGFP-tagged pseudorabies virus was used to enable in vitro electrophysiological analysis of infected neurons labeled transynaptically in vivo, demonstrating a potential use in tracing CNS circuitry. This could potentially be used to monitor spread through the CNS for MHV tagged with EGFP. Also, the ability to isolate EGFP-expressing cells infected with MHV makes it feasible to use fluorescence-activated cell sorting to determine the cell types infected by MHV.

\section{Materials and methods}

\section{Virus and cells}

FCWF cells and fMHV were obtained from Paul S. Masters (Albany, New York). fMHV is a recombinant $\mathrm{MHV}$ that contains the ectodomain of the $\mathrm{S}$ protein of feline infectious peritonitis virus, with the rest of the genes derived from MHV-A59 (Kuo et al, 2000). Parental viruses are $\mathrm{S}_{\mathrm{A} 59} \mathrm{R} 16$, recombinant $\mathrm{MHV}-\mathrm{A} 59$, and $\mathrm{S}_{4} \mathrm{R} 22$, isogenic with $\mathrm{S}_{\mathrm{A} 59} \mathrm{R} 16$ except that it contains the MHV-4 spike gene (Phillips et al, 1999). These parental viruses will be referred to more simply as $\mathrm{S}_{\mathrm{A} 59} \mathrm{R}$ and $\mathrm{S}_{4} \mathrm{R}$, respectively. Viruses were propagated on either murine 17Cl-1 cells or feline FCWF cells, and plaque assays and purifications were carried out on murine L2 cells. Cells were maintained on plastic tissue culture flasks in Dulbecco's minimal essential medium (DMEM) with $10 \%$ fetal bovine serum (FBS).

\section{Plasmids and PCR mutagenesis}

The pMH54 plasmid (obtained from Paul S. Masters) comprises codon 28 of the hemagglutinin esterase (HE) pseudogene through to the $3^{\prime}$ end of the MHVA59 genome, including a poly(A) tail, as described by Kuo et al (2000), used as transcription vector to generate RNA for targeted recombination as described previously (Phillips et al, 1999; Das Sarma et al, 2000). In order to replace gene 4 with the EGFP gene, pMH54 was modified by the introduction of a SalI site 42 nucleotides downstream of the intergenic sequence for gene $4 \mathrm{a}$ and a NotI site $102 \mathrm{bp}$ upstream of the stop codon for gene $4 \mathrm{~b}$, using the Quick Change site-directed mutagenesis kit (Stratagene, La Jolla, CA). (These are coding-silent nucleotide changes.) The coding sequence of EGFP was cleaved from the pEGFP-N1 vector (Clontech, Palo Alto, CA) using SalI and NotI and inserted in the place of the SalI/NotI fragment of pMH54. The resulting plasmid contains $760 \mathrm{bp}$ of non-MHV sequence, including the 722-bp EGFP open reading frame, replacing the entire gene $4 \mathrm{a}$ and the first $213 \mathrm{bp}$ of gene $4 \mathrm{~b}$. Then, to insert the EGFP fragment into the original unmodified pMH54 plasmid, a 1450-bp fragment of the EGFP-containing pMH54 was cleaved with SbfI (after the stop codon of the spike gene) and EcoRV at the $3^{\prime}$ end of ORF 5b, the E gene, and inserted it into the corresponding sites in pMH54; the final plasmid was called pMH54 $4_{\text {EFFP. }}$ Finally, the 1450-nucleotide SbfI/EcoRV fragment of this construct was sequenced (Sanger et al, 1977). To construct a similar plasmid differing only in the spike gene (called pMH54-S4EGFP), the MHV-4 spike gene was cleaved with AvrII and SbfI from pGEM-S4 (Phillips et al, 1999) and inserted into pMH54 in place of the A59 spike gene. For RNA transcription, the designated pMH54-derived plasmids were linearized just $3^{\prime}$ to the poly(A) tail by digestion with PacI. The EGFP gene/viral junctions were sequenced in both pMH54 EGFP and pMH54-S4EGFP using the primers FIJ81, FIJ83, RIJ84, and RIJ86 (Table 1) and the Taq dye terminator procedure (Taq DyeDeoxy Terminator Cycle Sequencing Kit; Applied Biosystems, Foster City, CA).

\section{Targeted $R N A$ recombination}

Targeted RNA recombination was carried out between synthetic capped RNAs transcribed from pMH54 EGFP or pMH54-S4EGFP, using a T7 polymerase transcription kit (Ambion, Austin, TX) and fMHV as a recipient virus. Recombinant viruses in which the murine coronavirus spike gene has replaced the feline coronavirus spike gene were selected by replication in murine 17Cl-1 cells (Phillips et al, 2001; Kuo et al, 2000). Candidate recombinants were plaque purified two times, and viral stocks were grown on $17 \mathrm{Cl}-$ 1 cells for further characterization. For each desired recombinant, at least two viruses derived from independent recombination events were characterized. The viruses with the A59 spike were called $S_{\text {A59 }} R_{\text {EGFP }}$ and those with the MHV-4 spike were called $\mathrm{S}_{4} \mathrm{R}_{\mathrm{EGFP}}$. Although each individual virus was given a number, such as $\mathrm{S}_{\mathrm{A} 59} \mathrm{R}_{\mathrm{EGFP}-1}$ or $\mathrm{S}_{4} \mathrm{R}_{\mathrm{EGFP}-1}$, they will be referred to here more simply as $S_{A 59} R_{E G F P}$ or $\mathrm{S}_{4} \mathrm{R}_{\mathrm{EGFP}}$.

\section{Virus growth curves}

Confluent monolayers of L2 cells (in DMEM with $10 \%$ FBS) were infected with each virus (1 PFU/cell) in triplicate and incubated for $1 \mathrm{~h}$ at $37^{\circ} \mathrm{C}$. Following adsorption, the cells were washed with Tris-buffered saline three times and then fed with DMEM-10\% FBS. At the times indicated, the cells were lysed by 
three cycles of freeze-thawing, and the virus in the lysates titered by plaque assay on L2 cells as previously described (Gombold et al, 1993)

\section{Northern blot analysis}

Total cellular RNA was isolated from infected (or mock-infected) L2 cells at $10 \mathrm{~h}$ post infection, and electrophoresed in 1\% agarose-formaldehyde gels (12 $\mu \mathrm{g}$ per lane). Gels were blotted onto Nytran SuPerCharge Nylon and hybridized for $2 \mathrm{~h}$ with $\left[\alpha-{ }^{32} \mathrm{P}\right] \mathrm{dCTP}-\mathrm{labeled}$ DNA probes. Probes were labeled using the Ready To Go DNA labeling beads (Pharmacia, Peapack, NJ) in which DNA was randomly primed. Template for the EGFP probe was NotI-digested EGFP-N1 plasmid (Clontech, Palo Alto, CA), DNA and template for nucleocapsid probe was PstI-digested pGEM 320 (Gombold and Weiss, 1992). The membranes were analyzed and bound probes were visualized using a phosphorimager (BioRad, Hercules, CA).

\section{Protein immunoblot analysis}

L2 cells were mock-infected or infected with either wild-type MHV-A59 or MHV-4, parental recombinant viruses $\mathrm{S}_{\mathrm{A} 59} \mathrm{R}$ or $\mathrm{S}_{4} \mathrm{R}$, or the EGFP-expressing viruses $\mathrm{S}_{\mathrm{A} 59} \mathrm{R}_{\mathrm{EGFP}}$ and $\mathrm{S}_{4} \mathrm{R}_{\mathrm{EGFP}}$ at a multiplicity of $1 \mathrm{PFU}$ per cell. At $12 \mathrm{~h}$ post infection, cells were lysed with $100 \mu \mathrm{l}$ of $10 \mathrm{mM}$ Tris-HCl, $\mathrm{pH}$ 7.5, $150 \mathrm{mM} \mathrm{NaCl}$, $1 \mathrm{mM}$ EDTA, $0.25 \%$ Nonidet P-40, complete Protease Inhibitor Cocktail Tablets (Roche, Mannheim, Germany) and $0.01 \%$ sodium azide for at $4{ }^{\circ} \mathrm{C}$, and clarified by centrifugation. Cell lysates $(25 \mu \mathrm{g})$ were electrophoresed in $10 \%$ SDS-polyacrylamide gels, which were then transferred to PVDF membranes (in $50 \mathrm{mM}$ Tris, $380 \mathrm{nM}$ glycin, $0.025 \%$ SDS, 20\% $\mathrm{MeOH})$. The membranes were blocked overnight using blotto $(40 \mathrm{mM}$ Tris, $5 \%$ [w/volt] Carnation powdered milk, and $0.01 \%[v / v]$ Tween-20), incubated for $2 \mathrm{~h}$ with either anti-nucleocapsid protein monoclonal antibody (clone 1-16-1 obtained from Dr. Julian Leibowitz, Texas A\&M University) at a dilution of $1 / 100$ or anti-GFP polyclonal antisera (Molecular Probe, Eugene, OR) at a dilution of $1 / 3000$, followed by $1 \mathrm{~h}$ incubation of horseradish peroxidase (HRP)-conjugated goat antimouse IgG (Roche, Mannheim, Germany) at a dilution of 1/2000 and HRP-conjugated goat anti-rabbit IgG (Jackson Immunoresearch, West Grove, PA) at a dilution of $1 / 10000$. The immunoblots were washed in phosphate-buffered saline (PBS) and then visualized using enhanced chemiluminescence (ECL; Amersham, Piscataway, NJ).

\section{Animals}

Four-week-old, MHV-free C57Bl/6 (B6) male mice (National Cancer Institute, Frederick, MD) were used in all experiments. Mice were anesthetized with isoflurane (IsoFlo; Abbott Laboratories, North Chicago, IL) prior to inoculation. For intracranial inoculations, $25 \mu \mathrm{l}$ of diluted virus (in PBS contain- ing $0.75 \%$ bovine serum albumin) was injected onto the left cerebral hemisphere. Mock-infected controls were inoculated similarly but with an uninfected cell lysate.

\section{Replication and histology in mice}

The efficiency of replication of the recombinant viruses was determined in mice inoculated intracranially at the designated dose. On days $1,3,5$, and 7 post infection, mice were sacrificed, perfused with $10 \mathrm{ml}$ of PBS, and the brains and livers were removed. The left half of the brain and most of the liver were placed directly into $2 \mathrm{ml}$ of isotonic saline with $0.167 \%$ gelatin (gel saline). (The rest of the brain and liver were used for histology.) All organs were weighed and stored frozen at $-80^{\circ} \mathrm{C}$ until titered for virus. Brains were homogenized, and virus titers were determined by plaque assay on L2 cell monolayers (Phillips et al, 1999).

For analysis of EGFP fluorescence, frozen sections had to be used rather than paraffin-embedded sections, because paraffin-embedded sections exhibited high amounts of autofluorescence. Thus, the rest of the brain (right half) and liver fragments from infected mice (see above) were fixed overnight in PBS with $4 \%$ paraformaldehyde. Tissues were subsequently washed for $1 \mathrm{~h}$ in PBS, $4 \mathrm{~h}$ in $5 \%$ sucrose, and then overnight in $20 \%$ sucrose, then embedded in OCT medium (Tissue Tek) and sectioned sagitally with a microtome to $4 \mu \mathrm{m}$ thickness and mounted on slides. Some sections were stained with H\&E while others were left unstained for EGFP detection. For EGFP autofluorescence sections were fixed in ice-cold 95\% ethanol for $20 \mathrm{~min}$, incubated at room temperature, in ice-cold PBS for $10 \mathrm{~min}$, and then mounted on $4.8 \%$ MOWIOL in $50 \%$ glycerol. Slides were visualized by fluorescence microscopy using an Olympus X-70 microscope system with a $10 \times$ long-working-distance UPlanFl phase objective ( $0.3 \mathrm{na})$ and a filter pack suitable for EGFP (U-MWIBA BP460-490 | DM505 | BA515-550) fluorescence. Images were acquired with a Hammatzu Orca-1 CCD camera and Image Pro image analysis software (Media Cybernetics, Silver Spring, MD). To create a composite image of a tissue section, partially overlapping images were obtained from sequential fields and then manually combined to form a complete image with Adobe Photoshop. To quantify the level of EGFP expression, regions of the image containing pixel values between 128 and 255 were automatically identified using Image Pro, subject to a threshold maximum area/feature to avoid quantifying autofluorescent regions, such as folds in the tissue slice. The sum total of pixels in this intensity range was then obtained using Image Pro. The intensity range was then increased to a broader range (25 to $255)$ to enable the software to identify the entire tissue slice as a region of interest to get the total area of the tissue slice in pixels. The relative level of expression was then calculated as EGFP pixels/total pixels for a given image. 
For assessment of the demyelination, mice were infected intracranially with $5 \times 10^{4}$ PFU per mouse. At 30 days postinfection, infected mice were sacrificed, perfused with PBS, followed by $10 \%$ phosphate-buffered formalin. Spinal cords were removed, embedded in paraffin, and sectioned for staining with Luxol fast blue to detect plaques of

\section{References}

Bond CW, Leibowitz JL, Robb JA (1979). Pathogenic murine coronaviruses. II. Characterization of virus-specific proteins of murine coronaviruses JHMV and A59V. Virology 94: $371-384$.

Cormack BP, Valdivia RH, Falkow S (1996). FACSoptimized mutants of the green fluorescent protein (GFP). Gene 173: 33-38.

Das Sarma J, Fu L, Tsai JC, Weiss SR, Lavi E (2000). Demyelination determinants map to the spike glycoprotein gene of coronavirus mouse hepatitis virus. J Virol 74: 92069213.

Fischer F, Stegen CF, Koetzner CA, Masters PS (1997). Analysis of a recombinant mouse hepatitis virus expressing a foreign gene reveals a novel aspect of coronavirus transcription. J Virol 71: 5148-5160.

Gombold JL, Hingley ST, Weiss SR (1993). Fusion-defective mutants of mouse hepatitis virus A59 contain a mutation in the spike protein cleavage signal. J Virol 67: 45044512.

Gombold JL, Weiss SR (1992). Mouse hepatitis virus A59 increases steady state levels of MHC mRNAs in primary glial cell cultures and in the murine central nervous system. Microb Pathogen 13: 493-505.

Haas J, Park EC, Seed B (1996). Codon usage limitation in the expression of HIV-1 envelope glycoprotein. Curr Biol 6: $315-324$.

Houtman JJ, Fleming JO (1996). Dissociation of demyelination and viral clearance in congenitally immunodeficient mice infected with murine coronavirus JHM. J NeuroVirol 2: 101-110.

Kuo L, Godeke GJ, Raamsman MJ, Masters PS, Rottier PJ (2000). Retargeting of coronavirus by substitution of the spike glycoprotein ectodomain: Crossing the host cell species barrier. J Virol 74: 1393-1406.

Lavi E, Gilden DH, Wroblewska Z, Rorke LB, Weiss SR (1984). Experimental demyelination produced by the A59 strain of mouse hepatitis virus. Neurology 34: 597603.

Lavi E, Murray EM, Makino S, Stohlman SA, Lai MMC, Weiss SR (1990). Determinants of coronavirus MHV pathogenesis are localized to $3^{\prime}$ portions of the genome as determined by ribonucleic acid-ribonucleic acid recombination. Lab Invest 62: 570-578.

Leparc-Goffart I, Hingley ST, Chua MM, Jiang X, Lavi E, Weiss SR (1997). Altered pathogenesis of a mutant of the murine coronavirus MHV-A59 is associated with a Q159L amino acid substitution in the spike protein. Virology 239: 1-10.

Leparc-Goffart I, Hingley ST, Chua MM, Phillips J, Lavi E, Weiss SR (1998). Targeted recombination within the spike gene of murine coronavirus mouse hepatitis demyelination. Demyelination was quantified by examining one spinal cord section (four quadrants) from each of five levels of spinal cord for each mouse; thus, approximately 100 quadrants were examined for each virus. Slides were coded and read in a blinded fashion (Leparc-Goffart et al, 1997).

virus-A59: Q159 is a determinant of hepatotropism. $J$ Virol 72: 9628-9636.

Luytjes W, Bredenbeek PJ, Noten AF, Horzinek MC, Spaan, WJM (1988). Sequence of mouse hepatitis virus A59 mRNA 2: indications for RNA recombination between coronaviruses and influenza $\mathrm{C}$ virus. Virology 166: 415422.

Matthews AE, Weiss SR, Shlomchik MJ, Hannum LG, Gombold JL, Paterson Y (2001). Antibody is required for clearance of infectious murine hepatitis virus A59 from the central nervous system, but not the liver. J Immunol 167: 5254-5263.

Navas S, Seo SH, Chua MM, Sarma JD, Lavi E, Hingley ST, Weiss SR (2001). Murine coronavirus spike protein determines the ability of the virus to replicate in the liver and cause hepatitis. J Virol 75: 2452-2457.

Ontiveros E, Kuo L, Masters PS, Perlman S (2001). Inactivation of expression of gene 4 of mouse hepatitis virus strain JHM does not affect virulence in the murine CNS. Virology 289: 230-238.

Phillips JJ, Chua MM, Lavi E, Weiss SR (1999). Pathogenesis of chimeric MHV4/MHV-A59 recombinant viruses: The murine coronavirus spike protein is a major determinant of neurovirulence. J Virol 73: 7752-7760.

Phillips JJ, Chua M, Seo SH, Weiss SR (2001). Multiple regions of the murine coronavirus spike glycoprotein influence neurovirulence. J NeuroVirol 7: 421-431.

Sanger F, Nicklen S, Coulson AP (1977). DNA sequencing with chain-terminating inhibitors. Proc Natl Acad Sci USA 74: 5463-5467.

Schwarz B, Routledge E, Siddell SG (1990). Murine coronavirus nonstructural protein ns2 is not essential for virus replication in transformed cells. J Virol 64: 4784-4791.

Slifka MK, Pagarigan R, Mena I, Feuer R, Whitton JL (2001). Using recombinant coxsackievirus $\mathrm{B} 3$ to evaluate the induction and protective efficacy of CD8+ T cells during picornavirus infection. J Virol 75: 2377-2387.

Smith BN, Banfield BW, Smeraski CA, Wilcox CL, Dudek FE, Enquist LW, Pickard GE (2000). Pseudorabies virus expressing enhanced green fluorescent protein: a tool for in vitro electrophysiological analysis of transsynaptically labeled neurons in identified central nervous system circuits. Proc Natl Acad Sci USA 97: 9264-9269.

Sutherland RM, Chua MM, Lavi E, Weiss SR, Paterson Y (1997). CD4+ and CD8+ T cells are not major effectors of mouse hepatitis virus A59-induced demyelinating disease. J NeuroVirol 3: 225-228.

Yokomori K, Lai MMC (1991). Mouse hepatitis virus S sequence reveals that nonstructural proteins ns4 and ns5a are not essential for murine coronavirus replication. J Virol 65: 5605-5608. 